(C) 2017 IEEE. Personal use of this material is permitted. Permission from IEEE must be obtained for all other uses, in any current or future media, including reprinting/republishing this material for advertising or promotional purposes, creating new collective works, for resale or redistribution to servers or lists, or reuse of any copyrighted component of this work in other works. 


\title{
The impact on Full Duplex D2D Communication of different LTE Transmission Techniques
}

\author{
Noman Haider ${ }^{1}$, Eryk Dutkiewicz ${ }^{1}$, Diep N. Nguyen ${ }^{1}$, Markus Mueck $^{2}$, Srikathyayani Srikanteswarae ${ }^{3}$ \\ ${ }^{1}$ University of Technology Sydney, Global Big Data Technology Centre, Ultimo NSW 2007, Australia. \\ 2Intel, Europe. \\ ${ }^{3}$ Intel, USA.
}

\begin{abstract}
To augment capacity of spectrum limited cellular systems, 3GPP proposed Licensed Assisted Access (LAA-LTE) while efforts are underway to standardize the standalone MulteFire (a small cell standalone version of LTE). LAA is expected to boost capacity of LTE via unlicensed spectrum (5GHz). On the other hand, recent advances in Self Interference Suppression (SIS) techniques allow radios to transmit and receive simultaneously on the same channel (i.e., in-band FullDuplex, FD). As part of future wireless networks, Device-todevice (D2D) communications would find its great potential through this FD capability. However, due to high induced aggregate interference from FD and its impact on medium access probability, the rigorous and critical analysis is needed to find an optimum trade-off between performance efficiency and overheads. Using stochastic geometry and the random graph theory, in this article, we analyze the impact of different LTE network paradigms with HD/FD D2D devices. Moreover, the impact of state-of-the-art coexistence techniques (discontinuous transmission and listen-before-talk) recommended for LTE in unlicensed spectrum over HD/FD D2D network is also discussed. The analysis is supported with extensive simulation results that reveal insights of the coexistence mechanism efficiency employed by LTE, the impact of SIS and the cost of FD operation in D2D.
\end{abstract}

Keywords-LTE-LAA, D2D, Coexistence technique, HalfDuplex, Full-Duplex modes, Self-Interference Suppression (SIS), unlicensed bands.

\section{INTRODUCTION AND RELATED WORK}

Recent years have witnessed an exponential growth of wireless data, largely coming from mobile broadband demand. Such a tremendous increase challenges network capacity, spectral efficiency and data rates. To meet the evergrowing demand, various technologies have been proposed to be included in the next generation (5G) mobile system [1] . For instance, due to scarcity of licensed spectrum and the advancements in dynamic spectrum access (DSA), mobile operators are eyeing on unlicensed spectrum $(5 \mathrm{GHz})$ and beyond [2]. New communications paradigms like device-todevice (D2D), vehicle to vehicle (V2V), etc have been also proposed. Additionally, at the physical layer, various advances have recently been made.

Dynamic spectrum allocation has brought forth a multifold increase in network capacity and throughput. Moreover, The latest developments in Self Interference Suppression (SIS) techniques enable in-band full-duplex (FD) communications in which a transceiver can transmit and receive simultaneously on the same frequency channel and even using the same antennas. That in-band FD capability has a potential of doubling the spectral efficiency of all existing wireless systems. However, with a trivial integration of FD into existing communications paradigms would result in excessive networkinterference [3]. In fact, the advantage of FD depends on whether SIS is perfect or imperfect as well as a meticulous network designs as discussed in [4]. Similarly, $\mathrm{D} 2 \mathrm{D}$ is also proven to be a potential candidate to augment the capacity of cellular networks [5], however, at the expense of introducing additional network interference. Different network models of cellular and D2D communications can be envisioned based on the centralized or distributed D2D communications scenarios.

The network's ultra-densification using small cells over unlicensed or shared spectrum with D2D connectivity is one of the key communications paradigm for 5G [1]. In this work, we consider the potential integration of FD capability to this scenario. We observe that due to the higher interference from in-band FD communications as well as D2D, FD-capable D2D devices may wish to opportunistically switch between Half-Duplex (HD) and FD modes. LTE and overlay FD D2D communications were studied with imperfect SIS and varying distance distributions between D2D nodes in [6]. The authors in this work used stochastic a geometry analysis to find the feasible network parameters and interference protection for LTE users.

The stochastic geometry and random graph theory has emerged as a powerful tool to study key network parameters[7]. Thanks to its mathematical tractability, it has been also used for analysis of heterogeneous networks (HetNets) [8] and the performance study of different coexistence techniques in spectrum sharing between LTELAA and WiFi [8]. A baseline model for throughput analysis of HD/FD systems and impact of SIS have recently been reported using stochastic geometry [3]. This analysis is further extended to model the performance of a LTE network with HD/FD D2D in the licensed bands [6]. However, to the best of our knowledge, none of these works have considered the impact of LTE in unlicensed bands with the legacy coexistence techniques for HD/FD D2D communications.

The coexistence between LTE-LAA and WiFi has been extensively investigated in recent works.. Coexistence techniques for LAA and WiFi include listen-before talk $(L B T)$ and Discontinuous Transmission (DTX). For instance, authors in [10] employed these methodologies along with Q-learning for an efficient co-existence of $\mathrm{WiFi}$ and LAA. The real-time test-bed results are also reported for $\mathrm{LAA}$ and $\mathrm{WiFi}$ 
coexistence in [11]. Stochastic geometry analysis of the stateof-the-art coexistence approaches for LAA and WiFi is presented in [8]. Although, these works provide good insights into the LTE and WiFi coexistence, they did not consider the impact of the LTE network on D2D performance and the HD/FD mode with different SIS

The existing literature provides a good insight for coexistence analysis between $\mathrm{WiFi}$ and LTE-LAA, however, the impact of state-of-the-art coexistence techniques on D2D nodes in unlicensed bands needs to be investigated. Moreover, the impact of SIS for FD D2D modes also need to be incorporated for critical analysis. This article presents detailed analysis of SIS effect and coexistence techniques used by LTE on D2D network on unlicensed bands using stochastic geometry.

The rest of the paper is organized as follows. The system model, assumptions, and different LTE transmission techniques are in Section II. Detailed numerical analysis, results and discussions are discussed in Section III. The conclusion of the paper is presented in Section IV.

\section{SySTEM MODEL}

\section{A. Spatial Locations}

Consider LTE small cells using unlicensed bands (LTELAA) that coexist with D2D nodes in the same vicinity. D2D nodes are equipped with FD capability and can operate in either HD or FD mode. LTE eNBs and UEs are assumed to operate in the HD. The LTE nodes are distributed according to an independent Poisson Point Process (PPP) $\Phi_{x}=\left\{x_{i}\right\}$ on the field $\mathrm{R}^{2}$, where the ground process $x_{i}$ is a PPP with intensity $\lambda_{L}$ that defines the set of locations of the LTE nodes. From the PPP property, the pdf of the cellular link distance is $f_{r}=2 \pi \lambda x e^{-\pi \lambda x 2}, x \geq 0$. Each UE is connected to its closest eNB, which provides the strongest average received power

D2D nodes's distribution follows another independent PPP $\Phi_{y}=\left\{y_{i}, m\left(y_{i}\right), s\left(y_{i}\right)\right\}$ with intensity $\lambda_{D}$, where $y_{i}$ are $m\left(y_{i}\right)$ set of locations of D2D nodes. For simplicity, we set $\| y$ $m(y) \|=R, \forall y \in \Phi_{y}$, i.e. $\mathrm{R}$ is the distance of all the links between a D2D pair. The marks $m(y)$ are receivers for FD D2D nodes and can also be represented as $m\left(y_{i}\right)=y_{i}+R\left(\cos \varphi_{i}, \sin \varphi_{i}\right)$, where the angles $\varphi_{i}$ are independently and uniformly distributed on $[0,2 \pi]$. The independent marks $m\left(\Phi_{y}\right)=\left\{m\left(y_{i}\right)\right.$, $\left.y_{i} \in \Phi_{y}\right\}$, which are also PPP of intensity $\lambda_{D}$. Whereas, $s\left(y_{i}\right)$ marks the independently chosen state of the D2D link between $m\left(y_{i}\right)$ and $y_{i} . s\left(y_{i}\right)$ takes values from $\{0,1\}$ representing the state of the link either HD or FD, respectively. The probability of a link being in state HD or FD is $p_{1}$ and $p_{2}$, respectively. The expected value for the FD state of the link is expressed as $E_{1 F D}=p_{i}$. The SIS in FD links is considered to be imperfect with a residual self-interference-to-power ratio (SIPR) $\beta$. The value of $\beta$ ranges from 0 to 1 . An example of the realization of considered LTE and D2D network nodes and coverage modeled through stochastic geometry is shown in Fig. 1.

\section{B. Propagation Assumptions}

The D2D devices and LTE nodes transmit with powers $P_{D}$ and $P_{L}$, respectively over a channel with bandwidth $B$. Since

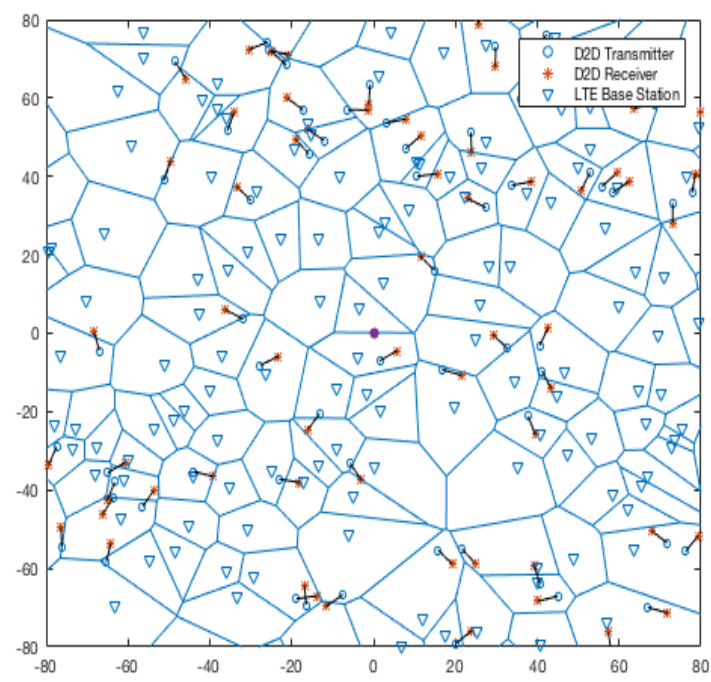

Fig. 1: Realization of network model with LTE and D2D nodes using Voronoi tessellation

D2D devices are communicating over a fixed distance of R, $P_{D}$ is significantly less than $P_{L}$. The path loss from a transmitter at $x$ to receiver at $y$ with distance $d$ is denoted by $l(d)$. The distance based path loss model is considered for propagation with the reference distance to the receiver denoted as ' $d$ '. The path loss attenuation factor is $\alpha$. Because, D2D links are mostly in LOS and collocated as compared to LTE nodes, the path loss of D2D links is less as compared with that of LTE links. All the channels are subject to i.i.d Rayleigh fading, with each fading variable $\left(F_{(y, x)}\right)$ exponentially distributed with parameter $\mu$.

\section{LTE Transmission Techniques}

This article considers three different scenarios based on the transmission method employed by LTE and studies its impact on FD D2D transmissions. In the first scenario, LTE transmits continuously without any protocol modifications and D2D pair communicates either in HD or FD mode with probability $p_{1}$ or $p_{2}$, respectively. When in the HD mode, the value of $\beta$ is varied to assess the impact of SIS for baseline results. In the second scenario, LTE uses a duty cycle based transmission pattern for $\eta$ fraction of time, whereas, the other devices in the medium transmit for $1-\eta$ time. In the third scenario, LTE devices use the LBT mechanism for channel access, where each node uses the aggregated energy detection (ED) in the medium and transmits only if the ED value is below a threshold. To implement LTE-LBT, PPP $\Phi_{x}=\left\{x_{i}, m\left(x_{i}\right)\right\}$ on $\mathrm{R}^{2}$ is considered, where each point $x_{i}$ is assigned with mark $m\left(x_{i}\right)$. This mark represents each point of the random back-off timer which is uniformly distributed between $(0,1)$. This back-off timer indicates the aggressiveness of LTE nodes for accessing the channel. Due to the difference in transmit powers and path loss, D2D nodes and the LTE nodes have different impacts on success probability.

Since both D2D and LTE nodes form a homogenous PPP, we perform our analysis by considering a receiver (y0) at the 
origin as it is equally applicable for all the other nodes in the network due to the motion invariance and translation property of PPP. The analysis is performed by considering full buffer downlink traffic only. Moreover, D2D nodes are always communicating whereas LTE nodes employ some coexistence mechanism to share the medium with incumbents. We denote the set of system parameters $\left(\lambda_{L}, \beta, \alpha\right)$ as the network configuration.

In the considered network scenario, a transmission attempt from all other nodes to a tagged node (at origin) is considered successful, if its SIR is greater than threshold T.

$$
\frac{S I R_{0}^{D}=}{\sum_{x \in \Phi x} P_{L} F_{0, x}^{L} l(0, x)+\sum_{y \in \Phi y}^{D} P_{D} F_{0, y}^{D} l(0, y)+\sum_{m(y) \in \Phi y} P_{D} F_{0, y}^{D} l(0, m(y)) 1_{y, m(y)}^{F D}+\beta P_{D} 1_{y, m(y)}^{F D}}
$$

\section{Success Probability}

For the system model discussed above, the performance metric is the transmission success probability for a D2D node located at the origin. The success probability is defined as,

$$
p_{s} \triangleq P\left(S I R_{y}>T\right)
$$

which can also be inferred as a complementary cumulative distributive function of SIR. If the SIR received at the tagged node is greater than threshold $T$, then the tagged node can successfully receive the desired signal. The success probability is a key metric that drives other network performance metrics i.e. throughput and data rate.

\section{RESULTS AND DISCUSSIONS}

The section presents performance impact of different LTE transmission types for D2D nodes operating in the HD and FD modes with different SIS values. The network configuration and simulation parameters, notations and values are given in Table I.

Fig. 2 characterizes the baseline performance for the success probability of the HD-only and FD-only modes. The FD-only network results in lower success probability as compared to the HD-only mode. We will further explore this

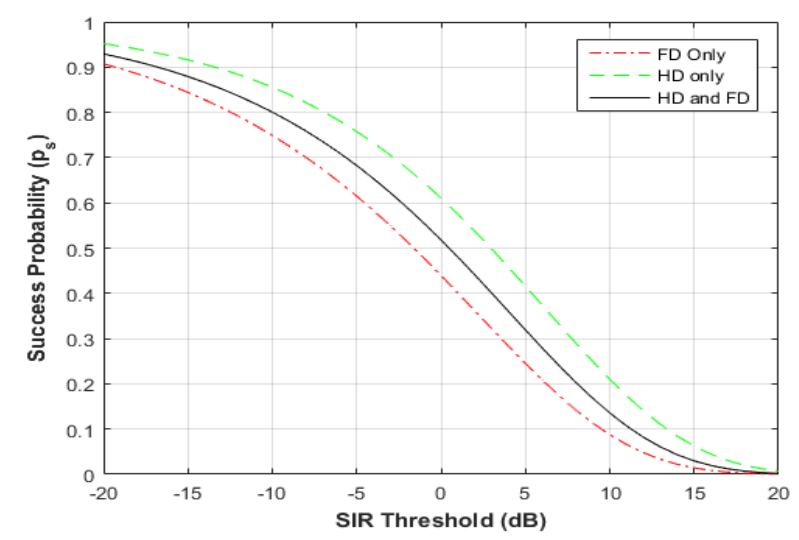

Fig. 2: Success probability of FD-only, HD-only and hybrid modes as a function of SIR threshold.
TABLE I. SIMULATION PARAMETER SETTING

\begin{tabular}{|c|c|}
\hline Parameters & Settings \\
\hline LTE transmit power $\left(P_{L}\right)$ & $1 \mathrm{~W}$ \\
\hline D2D transmit power $\left(P_{D}\right)$ & $0.005 \mathrm{~W}$ \\
\hline Path-loss component for D2D $\left(\alpha_{d}\right)$ & 3 \\
\hline Path-loss component for LTE $\left(\alpha_{L}\right)$ & $100 \mathrm{~m}$ \\
\hline Radius of circular area & Radius of area/20 m \\
\hline Link distance for D2D $(\mathrm{R})$ & 0.005 \\
\hline Self-Interference Cancellation $(\beta)$ & $2 * \lambda_{\mathrm{L}}$ \\
\hline LTE node intensity $\left(\lambda_{L}\right)$ & $-72 \mathrm{dBm},-77 \mathrm{dBm}$, \\
\hline D2D node intensity $\left(\lambda_{D}\right)$ & $-82 \mathrm{dBm}$ \\
\hline Energy Detection $(E D)$ & $0.33,0.5,0.7$ \\
\hline Duty Cycle $(\eta)$ & Exponentially \\
\hline distributed with $\mu$ \\
\hline Fading of channel from $x$ to $y\left(F_{(y, x)}\right)$ & 1 \\
\hline
\end{tabular}

result for different values of SIS to quantify the gains of the HD and FD mode.

\section{A. Continuous LTE Transmissions}

Fig. 3(top) compares the effect of LTE continuous transmission on the D2D success probability. It can be observed that by increasing the LTE intensity the performance of typical D2D pair degrades significantly. This behavior can be explained by the fact that the higher transmission intensity of the LTE nodes increases the interference floor for the D2D pair. To overcome this interference floor, the D2D pair should increase its transmit power for the successful transmission. However, increasing power is not a viable solution since it will affect the LTE users in return in the uplink. One possible solution to this problem is to shift the D2D transmission on to some other frequency band as mentioned by [9].

For this particular transmission technique, we compare the effect of SIS on D2D transmission in Fig. 3(bottom). Here the transmission intensity of LTE is taken as 0.005 . It can be observed from this figure that for the low SIR regimes there is much less effect of SIS on the successful reception. On the other hand, for higher SIR values the impact of SIS is prominent. So for D2D, in order to operate at higher SIR values, almost perfect SIS is required which can be achieved due to advancement of signal processing and chip designing techniques.

\section{B. LTE Discontinuous Transmissions (DTX)}

The simple method for LTE to ensure fairness and peaceful coexistence with technologies like WiFi is to employ duty-cycle based transmissions. To ensure fairness, the dutycycle can be adjusted according to the traffic conditions and the medium utilization by other technology candidates. Fig. 4 shows the impact of the LTE duty cycle on D2D success probability. As LTE transmit time increases from $33 \%$ to $75 \%$, the success probability degrades due to an increase in 

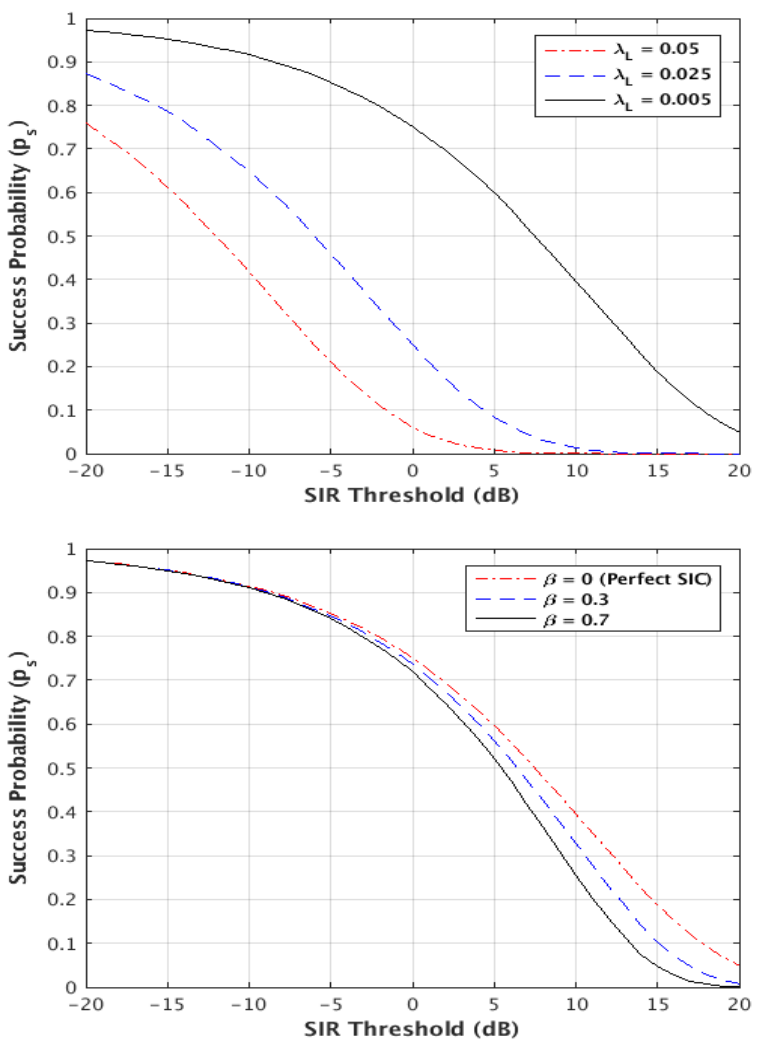

Fig. 3: Success probability of D2D nodes as a function of (top) SIS $\beta$ (bottom) LTE node intensity $\lambda_{\mathrm{L}}$

number of LTE nodes transmitting. As seen in the previous results, D2D network performance declines if LTE transmits for a longer period or the number of UEs tends to increase.

The LTE network does not guard or care for D2D communications as it transmits with the same power for $\eta$ fraction of time. Specially the LTE nodes transmitting in the vicinity of D2D communication degrade their performance. Therefore, from the results we conclude that the LTE nodes near the D2D nodes must use some sensing based mechanism to protect D2D nodes from strong interference.

\section{LTE Listen-before-Talk (LBT) with Contention Windown $(0,1)$}

Besides DTX, listen before talk (LBT) with a random back off is another coexistence mechanism based on energy detection in the medium. Each node has a random back off timer between $(0,1)$, which identifies how often the node senses the channel. Fig. 5(top) shows the impact of different energy detection thresholds (ED) for channel sensing on the success probability of the D2D nodes. For low ED values, the number of LTE nodes accessing the channel increases which in turns impacts the D2D nodes and their success probability decreases. An interesting observation from this result is that D2D communication is guarded by the LTE LBT transmission type. The LTE nodes in the vicinity of D2D pairs will guard D2D communication by not accessing the channel, however when the ED threshold is significantly reduced to $-10 \mathrm{dBm}$ then the success probability of the D2D nodes starts to drop.

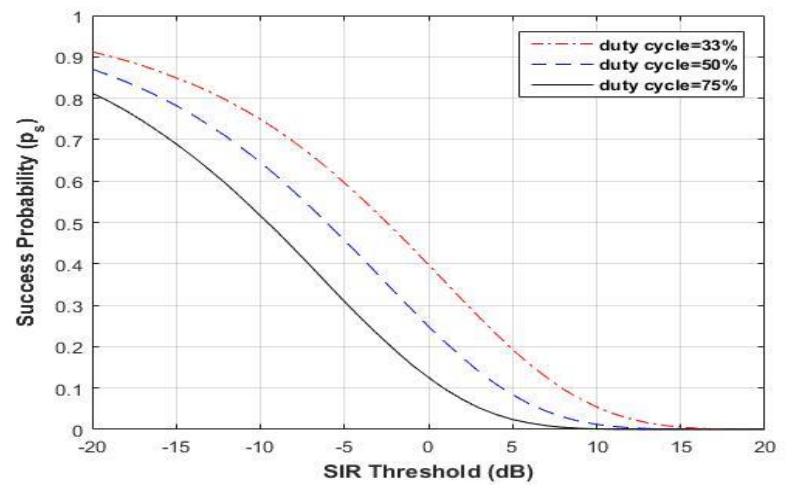

Fig. 4: The impact of LTE dutycycle $(\eta)$ on success probability of D2D nodes as a function of SIR threshold.
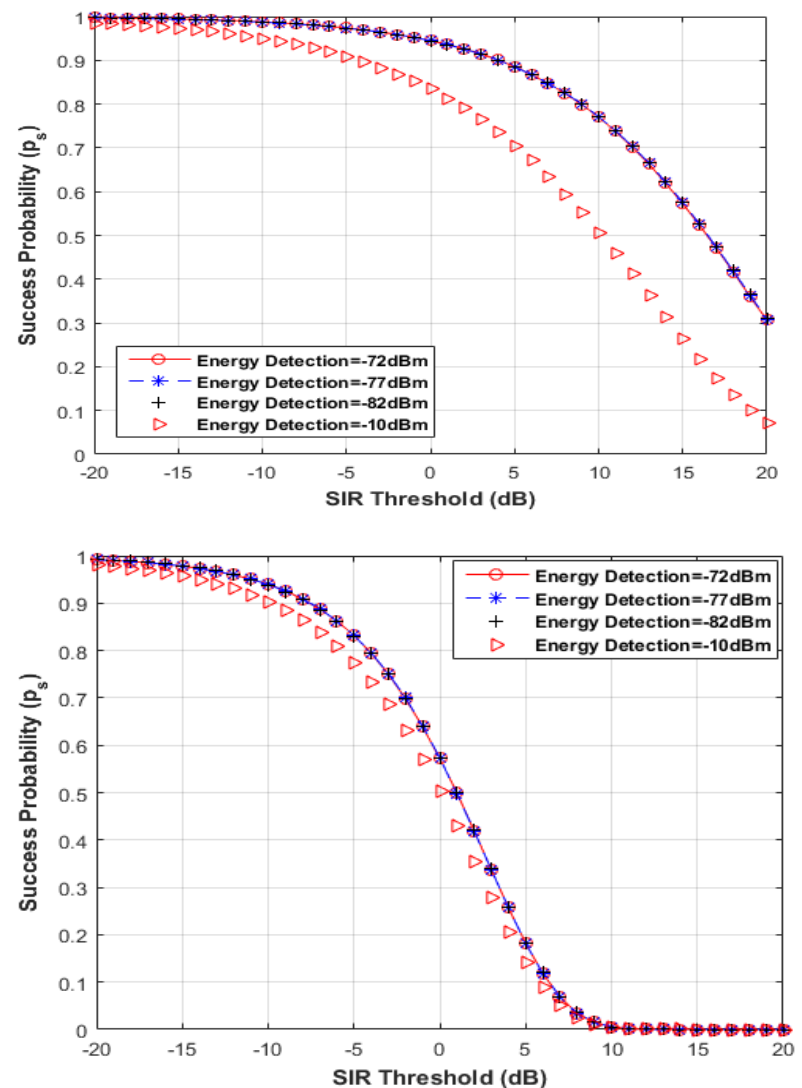

Fig. 5: The impact of LTE energy detection $(E D)$ threshld on success probability of D2D nodes as a function of SIR threshold with (top) $\beta=0$ (bottom) $\beta=0.8$

The other interesting results in Fig. 5 shows the impact of perfect $(\beta=0)$ and almost imperfect $(\beta=0.8)$ SIS when D2D is using the FD mode. Due to the increased residual SI, D2D communication suffers from severe interference from its own transmissions and from the LTE devices collectively. Therefore, the performance gain of FD communication is limited to the amount of SIS

Thus, LTE-LBT better guards D2D transmissions at the cost of the coverage outage of the nodes near D2D pairs. Based on mission-critical applications, D2D communication 
can be guarded by nearby LTE nodes and the optimum ED threshold can be selected to find the acceptable trade-off. The intuitive notion from the results indicate that although FD D2D may disrupt network performance due to an increased aggregate interference, performance gains can be achieved by carefully adjusting the power levels of the D2D and HD/FD mode selection for the D2D nodes. We aim to characterize this tradeoff between spectral efficiency, interference impact and network throughput in future work by using mathematical model.

\section{CONCLUSION}

In this article, the impact of different LTE transmission methods over HD and FD D2D networks is analyzed by using stochastic geometry analysis and simulations. The success probability for D2D nodes is numerically evaluated for scenarios when LTE nodes transmit continuously, employ discontinuous transmission or use the listen-before-talk mechanism. Moreover, the impact of Self-Interference Cancellation (SIS) on D2D success probability is evaluated. The LTE network is protected from D2D interference, whereas, the D2D success probability can be improved by carefully adjusting the transmit power and the modes of transmission. Also, near to perfect SIS results in a higher success probability for the D2D nodes without impacting the LTE network significantly. LTE-LBT better guards D2D pair communication by silencing the nearby nodes, however the energy detection threshold can be tuned accordingly to meet a tradeoff for success probability of D2D and LTE transmissions.

\section{ACKNOWLEDGMENT}

This work is supported in part by Intel's University Research Office.

\section{REFERENCES}

[1] I. F. Akyildiz, S. Nie, S.-C. Lin, and M. Chandrasekaran, "5G Roadmap: 10 Key Enabling Technologies," Computer Networks, 2016.

[2] A. Al-Dulaimi, S. Al-Rubaye, N. Qiang, and E. Sousa, "5G Communications Race: Pursuit of More Capacity Triggers LTE in Unlicensed Band," IEEE Vehicular Technology Magazine, vol. 10, pp. 43-51, 2015

[3] Z. Tong and M. Haenggi, "Throughput Analysis for Full-Duplex Wireless Networks With Imperfect Self-Interference Cancellation," IEEE Transactions on Communications, vol. 63, pp. 4490-4500, 2015.

[4] Diep N. Nguyen, Marwan Krunz, "Be Responsible: A Novel Communications Scheme for Full-Duplex MIMO Radios," Proc. of the IEEE INFOCOM Conference, Hong Kong, May 2015

[5] Y. Wu, W. Guo, H. Yuan, L. Li, S. Wang, X. Chu, et al., "Device-todevice meets LTE-unlicensed," IEEE Communications Magazine, vol. 54, pp. 154-159, 2016.

[6] K. S. Ali, H. ElSawy, and M.-S. Alouini, "Modeling Cellular Networks with Full Duplex D2D Communication: A Stochastic Geometry Approach," IEEE Transactions on Communications, vol. 64, no. 10, pp. 4409-4424, Oct. 2016.

[7] M. Haenggi, J. G. Andrews, F. Baccelli, O. Dousse, and M Franceschetti, "Stochastic geometry and random graphs for the analysis and design of wireless networks," IEEE Journal on Selected Areas in Communications, vol. 27, pp. 1029-1046, 2009.

[8] H. S. Dhillon, R. K. Ganti, F. Baccelli, and J. G. Andrews, "Modeling and analysis of K-tier downlink heterogeneous cellular networks," IEEE Journal on Selected Areas in Communications, vol. 30, pp. 550560, 2012.

[9] Y. Li, F. Baccelli, J. G. Andrews, T. D. Novlan, and J. C. Zhang, "Modeling and Analyzing the Coexistence of Wi-Fi and LTE in Unlicensed Spectrum," IEEE Transactions on Wireless Communications, vol. 15, no. 9, pp. 6310-6326, Sept. 2016.

[10] A. Galanopoulos, F. Foukalas, and T. Tsiftsis, "Efficient Coexistence of LTE with WiFi in the Licensed and Unlicensed Spectrum Aggregation," IEEE Transactions on Cognitive Communications and Networking, vol. PP, pp. 1-1, 2016.

[11] J. Yubing, S. Chao-Fang, B. Krishnaswamy, and R. Sivakumar, "Coexistence of Wi-Fi and LAA-LTE: Experimental evaluation, analysis and insights," in IEEE International Conference on Communication Workshop (ICCW), pp. 2325-2331, 2015 